kriegsverhütende, wie der Vertrag über die Nichtverbreitung von Kernwaffen vom 1. Juli 1968) und Beistandspakte. Dafür finden sich das bei Berber/Randelzhofer leider fehlende Internationale Ubereinkommen zur Beseitigung jeder Form von Rassendiskriminierung vom 7. März 1966, Minderheitenschutzverträge (wie das Gruber-De-Gasperi-Abkommen), eine Fülle seerechtlicher Verträge (auch zum marinen Umweltschutz, wie die Úbereinkommen von London und Oslo über Ocean Dumping), neutralitätsrechtliche Dokumente, die Konventionen von Den Haag, Tokio und Montreal zum Schutz des Luftverkehrs. Zu begrüßen ist auch die Aufnahme der Deklaration der Generalversammlung der Vereinten Nationen über ,Friedly Relations“ vom 24. Oktober 1970 und von Auszügen aus der Schlußakte der KSZE in Helsinki. Im Anhang finden sich Beispiele für Vorbehalte zu Verträgen, Anerkennungserklärungen und ähnliches. Auch in diesem Band sind jeweils deutsche Ưbersetzungen abgedruckt.

Beide Sammlungen erreichen ihr Ziel, rasche Orientierung zu ermöglichen, in zuverlässiger Weise. $\mathrm{Da}$ ihr Inhalt nur teilweise identisch ist, wird man häufig zu beiden greifen müssen.

Philip Kunig

\title{
BerTil Egero
}

\section{Colonization and Migration}

A Summary of border-crossing movements in Tanzania before 1967

Research Report No. 52 des Scandinavian Institute of African Studies, Uppsala, 1979, 45 S., Skr. 10,-

Es handelt sich um eine informative, sorgfältige und leicht lesbare Studie zu einem Gegenstand, der wegen seiner großen sozialen und ökonomischen Bedeutung besonderes Interesse von Wissenschaft und politischer Praxis beanspruchen kann. Die Untersuchung beginnt mit einer kurzen Schilderung der sozialen Rahmenbedingungen in Tansania vor und nach der Unabhängigkeit, soweit sie die Thematik betreffen. Egerö skizziert den ethnischen Ausgangspunkt und behandelt das Erscheinen und die Rolle der Araber, Europäer und Asiaten auf der ostafrikanischen Szene, schließlich die kontinentalen Wanderungsbewegungen der Afrikaner, dabei Einwanderung nach und Auswanderung aus Tansania. Das Thema legt an sich eine Auseinandersetzung mit den Ursachen der Migration nahe, die über die Nennung der historischen Fakten und Daten hinausginge. Dies kann freilich auf 40 Seiten nicht geschehen. Egerös „,Summary“ verdient Beifall für die klare Darstellung und übersichtliche Zusammenfassung. Seine Einschätzung der Situation in Tansania ist fair und kenntnisreich.

Costa R. Mahalu

\section{Zahra FreEth/Victor W INSTONE}

\section{Explorers of Arabia from the Renaissance to the Victorian Era.}

George Allen \& Unwin Ltd., London 1978, 308 S.

Das Buch dürfte nicht nur Orientalisten, sondern auch den allgemein interessierten Leser ansprechen. Die Verfasser beschreiben die Reisen von neun weniger bekannten Entdeckern auf der arabischen Halbinsel; die Wiedergabe der Reisen erfolgt unter Einschiebungen aus den Originalberichten und ist so plastisch, daß sie den Leser noch heute gefangen nimmt. Die Aufzeichnungen beginnen mit Varthema, der Anfang des 16. Jahrhunderts von Ägypten kommend als erster Europäer bis Mekka und Medīna gelangte. In namhaften Nachschlagewerken wird diese seinerzeit nur durch ungeheure Mühsal erreichte Ehre J. L. Burckhardt zugeschrieben - einem auch hier verzeichneten Reisenden, der sich um die Wiederentdek- 
kung von Petra verdient gemacht hat. Die Verfasser zeichnen das in der algerischen und tunesischen Sklaverei erlittene Schicksal von Pitts, seine Reisen mit seinem muslimischen Herrn nach Mekka und Medīna ebenso auf wie die Yemen-Reise des Dänen Niebuhr, der als einziger von sechs Reisegenossen überlebte, und die Reisen ins Nafūd und Nağd von Burton, der durch seine Aufzeichnungen und die Ubersetzung von 1001 Nacht ins Englische unmittelbaren Ruhm erlangte. Der letzte Reisebericht ist dem Ehepaar Blunts gewidmet, das 1879 von Damaskus aus nach Hail reiste; Lady Anne ist die erste Europäerin, die so weit in arabisches Gebiet vordrang. Die wohl schillerndste Gestalt ist der Engländer Gifford Palgrave. Jüdischer Abstammung, von der Familie für den Staatsdienst vorgesehen, meldet er sich zur Armee und geht nach Indien, nimmt den Katholizismus an, tritt aus der Armee aus und wird 1856 als Jesuit zum Priester geweiht; nach seinen Reisen verfaßt er in einem deutschen Kloster ein Buch über seine Erlebnisse in al-Riyadh, dem Zentrum der WahhabitenBewegung, tritt dann aus dem Jesuitenorden aus und beschließt sein Leben als Mitglied des diplomatischen Korps. Bemerkenswert ist auch das Verhalten von Doughty, der in den Jahren von 1876 bis 1878 wie Burton das Nafüd und Nağd bereiste, sich wie ein Beduine gerierte, auf Befragen nach seiner Herkunft jedoch stets seine christliche Úberzeugung auch gegenüber konservativ denkenden Wahhabiten kundtat und dadurch mehrfach und zusätzlich zu den ohnehin bestehenden Fährnissen in Lebensgefahr geriet.

Dagmar Hohberger

Helmut Martin

\section{Kult und Kanon}

Entstehung und Entwicklung des Staatsmaoismus 1935-1978

Mitteilungen des Instituts für Asienkunde in Hamburg, Nr. 99, 1978, 101 S., 15 DM

In dieser Schrift legt der Sinologe Helmut Martin Arbeitsergebnisse vor, die aus dem von ihm geleiteten, großangelegten Projekt einer deutsch-chinesischen Ausgabe der Schriften, Reden, Gespräche Mao Tse-tungs ${ }^{1}$ resultieren. Den ,'Staatsmaoismus“" konstituieren für Martin ,,Mao-Kult“ und ,,-Kanon“; die Entwicklung des letzteren verfolgt er von den 1937 erschienenen ,,Abhandlungen Mao Tse-tungs“ als Vorläufer der berühmten späteren ,,Ausgewählten Werke" bis hin zu den Auseinandersetzungen um das Erscheinen und den Inhalt von deren fünftem Band (erschienen im April 1977), in dessen Mittelpunkt die Rede Maos „Über die zehn großen Beziehungen“" von 1956 steht: im Gegensatz zu vielen anderen, typischen Texten ausgezeichnet geeignet, das politische Primärziel der Deng und Hua, den ,,Aufbau", als auch von Mao mit gleicher Intensität verfolgt darzustellen. Es ist Martin gelungen, die Wandlungen der Editionspolitik betreffend Maos Schriften straff darzustellen und sie jeweils vor dem Hintergrund der Entwicklung von Partei und Staat insbesondere im Nachkriegschina zu erläutern. Den Geschehnissen nach 1977 ist dabei der relativ größte Raum gegeben. Eine Spekulation über die zukünftige Rolle der Verehrung für Mao und die Bedeutung seiner Gedanken in der Volksrepublik China - die für Lin Biao (1976) den ,,Zenit des Marxismus-Leninismus unserer Zeit" darstellten - wagt Martin nicht; in der Tat dürften sich heute kaum plausible Prognosen darüber abgeben lassen, ob Mao-Statuen gleich solchen Stalins dereinst auf dem Trümmerhaufen der Geschichtelanden, ob Kräften erneut in China ein Aufstieg gelingen kann, die seine Texte wieder mehr im Sinne des historischen Mao kanonisieren werden, oder wo zwischen diesen Polen das Vermächtnis des Großen Steuermannes seinen Platz wird zugewiesen erhalten.

Philip Kunig

1 Die auf sechs Textbände und einen Kommentar- und Registerband angelegte Ausgabe („,Mao Zedong, Texte 1949-1967“) ist seit 1978 im Erscheinen; sie soll im Herbst 1980 vollständig vorliegen. 